\title{
Die 5-Item-Skala zur Messung der momentan verfügbaren Selbstkontrollkapazität (SMS-5) im Lern- und Leistungskontext
}

\section{Eine Validierungsstudie}

\author{
Christoph Lindner ${ }^{1,2} \odot$, Marlit Annalena Lindner ${ }^{1}$ und Jan Retelsdorf ${ }^{3}$ \\ 'Leibniz-Institut für die Pädagogik der Naturwissenschaften und Mathematik (IPN) Kiel \\ ${ }^{2}$ Christian-Albrechts-Universität (CAU) zu Kiel \\ Universität Hamburg
}

\begin{abstract}
Zusammenfassung: In Leistungssituationen sind Personen angehalten Selbstkontrolle aufzuwenden, um ihre Aufmerksamkeit, Gedanken und Emotionen zielgerichtet zu regulieren. Selbstkontrolle wird als begrenzte mentale Kapazität betrachtet, die situativen Fluktuationen unterliegt und sich kurzfristig erschöpfen kann. In dieser Validierungsstudie stellen wir eine 5-Item-Skala zur Messung der momentan verfügbaren Selbstkontrollkapazität (SMS-5) basierend auf der State Self-Control Capacity Scale (SSCCS) vor, mit dem Ziel auch in kurzen zeitlichen Abständen intra- und interindividuelle Variabilitäten der momentan verfügbaren Selbstkontrollkapazität (d. h. die Ausprägung der empfundenen mentalen Erschöpfung) ökonomisch abzubilden und externe Kriterien im Lern- und Leistungskontext valide vorherzusagen. Die interne Struktur der SMS-5 erwies sich in Stichproben von Auszubildenden ( $N=2395)$, Zehntklässlerinnen und Zehntklässlern ( $N=129)$ und Studierenden $(N=95 ; N=140)$ als eindimensional. Zusammenfassend zeichnete sich die SMS-5 als valides, reliables, über Ausbildungsgruppen, Geschlecht und Zeit invariantes und änderungssensitives Instrument zur ökonomischen Messung der momentan verfügbaren Selbstkontrollkapazität im Lern- und Leistungskontext ab, das gegenüber der SSCCS in der Vorhersage externer Kriterien vergleichbar gut abschnitt. Die SMS-5 empfiehlt sich besonders, um durch kurzfristig wiederholte Messungen, Veränderungen der Selbstkontrollkapazität im zeitlichen Verlauf von Lern- und Leistungshandlungen abzubilden.
\end{abstract}

Schlüsselwörter: Selbstkontrolle, Selbstregulation, Mentale Erschöpfung, Ego Depletion Kurzskala, Fragebogen

Measuring Self-Control Depletion in Achievement Situations: A Validation of the 5-Item Brief State Self-Control Capacity Scale

Abstract: In achievement situations, individuals need to invest self-control in order to regulate their attention, thoughts, and emotions in a goal-directed manner. Research suggests that exerting self-control leads to decreasing self-control performance over time as a result of the short-term depletion of individuals' state self-control capacity. In four studies, we validated a new version - the 5-item scale for measuring the momentary self-control capacity (SMS-5; English version available in Table 1) - of Ciarocco's State Self-Control Capacity Scale (SSCCS) to assess changes in people's perceived state self-control capacity (i.e., degree of perceived mental exhaustion) in achievement situations. Drawing on German samples of apprentices in vocational education and training $(N=2,395)$, tenth-graders $(N=129)$, and university students $(N=95 ; N=140)$, we replicated the unidimensional internal structure of the SMS-5. Moreover, measurement invariance was supported for different groups of apprentices, for gender, and over time. The SMS-5 performed as well as the SSCCS in predicting achievement-related outcome variables and is a viable option for repeatedly assessing individuals' perceived state self-control capacity and for tracking mental exhaustion over time.

Keywords: self-control capacity, self-regulation, mental exhaustion, brief ego depletion scale, questionnaire

Als Voraussetzung für ein gutes Abschneiden in Lern- und Leistungssituationen gilt neben kognitiven Grundfähigkeiten die Ausübung von Selbstkontrolle, um die Aufmerksamkeit zielgerichtet und überdauernd auf aufgabenrele- vante Inhalte zu fokussieren und ablenkende Reize (z. B. irrelevante Gedanken) zu ignorieren. Es wird angenommen, dass dabei eine begrenzte mentale Kapazität - die Selbstkontrollkapazität - beansprucht wird, die für eine ef- 
fiziente Verhaltensadaptation notwendig ist und die Ausführung zielgerichteter Handlungen erst ermöglicht (z. B. Baumeister, Vohs \& Tice, 2007). Zahlreiche Befunde der psychologischen Grundlagenforschung (für einen Überblick siehe z.B. Hagger, Wood, Stiff \& Chatzisarantis, 2010) weisen darauf hin, dass die längerfristige Ausübung von Selbstkontrolle zu einer Abnahme der momentan verfügbaren Selbstkontrollkapazität und demnach zu einem kurzfristigen mentalen Erschöpfungszustand (Baumeister et al., 2007) führen kann (Ego-Depletion-Effekt), der sich von physiologischen Müdigkeitseffekten abgrenzen lässt (Lindner, Nagy, Ramos Arhuis \& Retelsdorf, 2017). In diesem Erschöpfungszustand gelingt es Personen vorübergehend schlechter, sich zu konzentrieren und ihre Aufmerksamkeit, Gedanken und Emotionen zu regulieren. So konnte beispielsweise gezeigt werden, dass sich infolge einer experimentell induzierten Selbstkontrollerschöpfung schlechtere Leistungen in kognitiven Tests einstellen, die schlussfolgerndes Denken oder eine starke Aufmerksamkeitsfokussierung erfordern (z.B. Friese, Messner \& Schaffner, 2012; Lindner et al., 2017; Schmeichel, Vohs \& Baumeister, 2003). Darüber hinaus wird auch die Regulation von leistungsbezogenen Ängsten und Stress kurzfristig beeinträchtigt (Bertrams, Englert, Dickhäuser \& Baumeister, 2013; Ciarocco, Twenge, Muraven \& Tice, 2007). Als Erklärung für diese Befunde wird angenommen, dass die Ausübung von Selbstkontrolle zu einer progredienten Abnahme in der mentalen Selbstkontrollkapazität und folglich zu einer zunehmenden Selbstkontrollerschöpfung führt, wodurch sich im zeitlichen Verlauf negative Selbstkontrollleistungen einstellen (Baumeister et al., 2007). Aktuellere Modelle nehmen hingegen an, dass die progrediente Abnahme in der Selbstkontrollkapazität auf die zeitliche Veränderung emotional-motivationaler Faktoren (Inzlicht \& Schmeichel, 2012) sowie auf eine Aufmerksamkeitsverschiebung (Inzlicht, Schmeichel \& Macrae, 2014) zurückzuführen ist. Die Abnahme der Selbstkontrollkapazität sollte demnach mit einer Zunahme eines negativen affektiven Zustandes einhergehen, wodurch eine motivierte Aufmerksamkeitsverschiebung in Richtung positiv assoziierter Stimuli wahrscheinlicher wird. Die Erschöpfung der Selbstkontrolle und die Performanzreduktion in Selbtkontrollaufgaben könnten somit Folge einer Anstrengungsreduktion bei der Bearbeitung kognitiver Aufgaben sein (Lindner, Nagy \& Retelsdorf, 2018). In diesem Zusammehang weisen Inzlicht und Schmeichel (2012) darauf hin, dass für die Untersuchung selbstkontrollbedingter Veränderungsprozesse beispielsweise Selbsteinschätzungsverfahren wiederholt eingesetzt werden sollten, um Prozessvariablen zu messen und Veränderungen im zeitlichen Verlauf abzubilden. Die Entwicklung von Fragebögen zur ökonomischen Messung von Selbstkontrollerschöpfungseffekten gewinnt zudem an Relevanz, da in einer groß angelegten, laborübergreifenden Studie von Hagger et al. (2016) der experimentell induzierte Ego-Depletion-Effekt nicht repliziert werden konnte. Hierbei ist jedoch anzumerken, dass die Validität der verwendeten selbstkontrollintensiven Manipulationsaufgabe in Frage steht und in der Literatur kritisch diskutiert wird (Baumeister \& Vohs, 2016; Lurquin \& Miyake, 2017). Davon ausgehend betonen Lurquin und Miyake (2017) den Bedarf von validen Instrumenten und alternativen Methoden zur Untersuchung von Selbstkontrollerschöpfungseffekten. Die Entwicklung eines ökonomischen Fragebogeninstruments zur Untersuchung der momentanen Selbstkontrollkapazität in Feldstudien erscheint als besonders hilfreich, da die Annahme besteht, dass Selbstkontrollerschöpfungseffekte sich erst nach längerer Zeit der Selbstkontrollaufwendung einstellen (Hagger et al., 2016). Es zeigte sich bereits in vier Studien von Clarkson, Hirt, Jia und Alexander (2010), dass besonders die bewusste Repräsentation der momentan verfügbaren Selbstkontrollkapazität prädiktiv für zeitlich naheliegende Selbstkontrollleistungen ist, unabhängig davon ob die Selbstkontrollkapazität im Vorfeld experimentell erschöpft wurde oder nicht. Diese Befunde weisen darauf hin, dass der Grad der Selbstkontrollerschöpfung bewusstseinsfähig ist. Damit einhergehend zeigen weitere Studien, dass die via Fragebogen gemessene momentane Selsbtkontrollkapazität prädiktiv für eine Vielzahl von Outcome-Variablen ist (z.B. Bertrams, Englert \& Dickhäuser, 2010; Schöndube, Bertrams, Sudeck \& Fuchs, 2017; Welsh \& Ordóñez, 2014).

Es besteht bereits ein umfangreicheres Fragebogeninstrument, anhand dessen die momentan verfügbare Selbstkontrollkapazität als domänenübergreifendes Konstrukt valide und breit erfasst werden kann. Genauer adaptierten Bertrams, Unger und Dickhäuser (2011) eine aus 25 Items bestehende Skala (die State Self-Control Capacity Scale Deutsch; SSCCS-D), sowie eine 10-Item-Kurzversion (SSCCS-K-D) aus dem Englischen (Ciarocco et al., 2007). Die Items beider Instrumente bestehen aus Aussagesätzen (vgl. Tabelle 2), die unter anderem das momentane Erleben von kognitiver Energie, der Erschöpfung von Willenskraft und der gegenwärtigen Konzentrationsfähigkeit widerspiegeln. Die psychometrische Güte beider eindimensionaler Skalen erwies sich als sehr gut (Bertrams et al., 2011). Die Instrumente von Bertrams et al. (2011) eignen sich, um Zusammenhänge zwischen der momentan verfügbaren Selbsktontrollkapazität und einer Vielzahl selbstkontroll-assoziierter Variablen aus den Bereichen Gesundheits-, Arbeits- und Leistungsverhalten vorherzusagen (z. B. Germeys \& De Gieter, 2018; Kühnel, Syrek \& Dreher, 2018; Lian, Yam, Ferris \& Brown, 2017). Auffällig ist jedoch, dass in einigen empirischen Studien trotz des Vorliegens der 10-Item SSCCS- $K$-D verschiedene SSCCS$D$-Item-Kompositionen eingesetzt wurden (z.B. Diestel, 
Rivkin \& Schmidt, 2015; Prem, Kubicek, Diestel \& Korunka, 2016; Rivkin, Diestel \& Schmidt, 2014, 2015; Rowland, Wenzel \& Kubiak, 2016), um Zusammenhänge der momentan verfügbaren Selbstkontrollkapazität und leistungsbezogenen Kriterien zu untersuchen. Dabei wurden häufig nur drei (z.B. Prem et al., 2016; Rowland et al., 2016) oder fünf Items der SSCCS-D (z. B. Diestel et al., 2015; Rivkin et al., 2014, 2015) eingesetzt, wobei die verwendeten Items über Autorengruppen variierten. Ähnliches gilt für die englische Originalskala (Ciarocco et al., 2007; z.B. Kwan, Bedard, King-Dowling, Wellman \& Cairney, 2016; Salmon et al., 2015). Dieser heterogene Itemeinsatz stellt die Vergleichbarkeit der Messungen und damit auch die Generalisierbarkeit berichteter Ergebnisse in Frage. Darüber hinaus liefern die Studien kaum Befunde zur psychometrischen Güte der verwendeten Items, wodurch die Qualität der Messungen uneindeutig ist. Diese Studien weisen darauf hin, dass trotz des Vorliegens der validen und reliablen SSCCS- $K-D$, offenbar der Bedarf für ein weitaus ökonomischeres Instrument zur spezifischen Messung der situativen Selbstkontrollkapazität besteht. Vor diesem Hintergrund war es unser Ziel, eine 5-ItemSkala zur Messung der momentan verfügbaren Selbstkontrollkapazität (SMS-5) basierend auf seligierten SSCCS-K-DItems zu entwickeln, die auch in kürzeren Zeitabständen wiederholt in Feldstudien eingesetzt werden kann, um Veränderungen in der momentanen Selbstkontrollkapazität (d.h.den Grad mentaler Erschöpfung) im Lern- und Leistungskontext valide abzubilden. Während die SSCCS$(K-) D$ die momentan verfügbare Selbstkontrollkapazität als domänenübergreifendes Konstrukt breiter erfasst und mit domänenübergreifenden externen Kriterien in Verbindung steht, war unser Anspruch eine Skala zu entwickeln, anhand derer die situative Selbstkontrollkapazität spezifisch im Lern- und Leistungskontext ökonomisch abgebildet werden kann. Trotz einer daraus möglicherweise folgenden Einschränkung in der Einsatzbreite, liegt der zentrale Vorteil der SMS-5-Kurzskala demnach in ihrer geringen Bearbeitungsdauer. Die Skala kann somit beispielsweise problemlos im Rahmen von Schulleistungstests, Intelligenztests oder Aufmerksamkeits-/Konzentrationstests wiederholt nach verschiedenen Aufgabenblöcken eingesetzt werden, ohne dabei den Testungsverlauf oder das Testverhalten zeitlich zu stark zu beeinflussen (Lindner et al., 2018). Des Weiteren sollte sich die $S M S-5$ auch für den Einsatz in Experience-Sampling-Studien eignen, mit dem Ziel interindividuelle Unterschiede und intraindividuelle Variabilitäten in der momentanen Selbstkontrollkapazität auch in Alltagssituationen zu untersuchen. Die Konzeption und Validierung unserer $S M S$-5-Kurzskala wird im Folgenden basierend auf vier unabhängigen Studien beschrieben.

\section{Der Itemselektionsprozess zur Erstellung der SMS-5}

Um die momentan verfügbare Selbstkontrollkapazität vor allem im Lern- und Leistungskontext ökonomisch abzubilden, haben wir diejenigen Items der SSCCS-K-D (Bertrams et al., 2011) in Erwägung gezogen, deren Formulierungen leistungsbezogene Aspekte selbstkontrollierten Verhaltens repräsentieren und von einer affektiven Bewertungsebene herrühren. Im Gegensatz zum experimentell induzierten Selbstkontrollerschöpfungseffekt, der anhand einer Performanzreduktionen in Selbstkontrollaufgaben operationalisiert wird, sollen die Items der SMS-5 das aktuelle Empfinden der Erschöpfung abbilden, welche durch das Ausüben von Selbstkontrolle entsteht (Baumeister et al., 2007). Inzlicht und Berkman (2015) sowie Hagger et al. (2016) verweisen darauf, dass eine situativ geringe Selbstkontrollkapazität eine Form mentaler Erschöpfung darstellt, weshalb die zu selegierenden Items der Kurzskala den affektiven Zustand der erlebten Erschöpfung durch das Aufwenden von Selbstkontrolle in Lern- und Leistungssituationen abbilden sollten. Unter Berücksichtigung von Trennschärfen und Faktorladungen in der Validierungsstudie der SSCCS-K-D von Bertrams et al. (2011) sowie der Urteile zweier Experten der Selbstkontrollforschung, haben wir fünf geeignete Items identifiziert (vgl. Tabelle 1) und zur SMS-5 zusammengestellt. Als geeignet wurden diejenigen Items befunden, die eine hohe psychometrische Güte in der Studie von Bertrams et al. (2011) aufwiesen, gleichzeitig aber vom Wortlaut weitestgehend zurückhaltend formuliert sind, sodass die Wahrscheinlichkeit einer Induktion reaktiven Verhaltens durch das Bearbeiten der Items minimal bleibt. Da Expertinnen und Experten aufgrund ihres Fachwissens die psychometrische Güte und den Wortlaut der Items gleichzeitig in ihren Auswahlprozess einbeziehen können, haben wir uns für diese Variante der Item-Selektion entschieden. Alle fünf Aussagesätze werden anhand einer siebenstufigen Ratingskala von $1=$,völlig unzutreffend“ bis $7=$,trifft ganz genau zu" bewertet. Analog zur SSCCS-K-D sind nur die Extremkategorien 1 und 7 zusätzlich verbal verankert, während den Kategorien 2 bis 6 nur nummerische Anker zugeordnet waren. Der Prompt der Skala sowie die Original Items von Bertrams et al. (2011) und Ciarocco et al. (2007) sind Tabelle 1 zu entnehmen.

\section{Studie 1}

Zunächst legten wir die Items der SMS-5 einer großen Stichprobe vor, um die psychometrische Güte der Skala basierend auf faktorenanalytischen Verfahren zu untersu- 
chen. Basierend auf Befunden von Bertrams et al. (2011) sowie konzeptuell-theoretischen Annahmen wurde eine eindimensionale faktorielle Struktur erwartet.

\section{Methode}

Stichprobe, Vorgehen und Analysen. Im Rahmen des Großprojektes „Mathematisch-naturwissenschaftliche Kompetenzen in der beruflichen Erstausbildung" (ManKobE, [SAW-2012-IPN-2]) wurde die SMS-5 N= 2395 Auszubildenden gewerblich-technischer (GTB), industriekaufmännischer (IK) und labor-technischer (LAB) Berufe (33\% weiblich; $M_{\text {Alter }}=18.43, S D_{\text {Alter }}=2.43$ ) in Hessen, Bayern und Baden-Württemberg vorgelegt. Zur Untersuchung der internen Struktur der SMS-5 wurde zunächst eine zufällige Substichprobe von $N=801$ (ca. 33\% der Gesamtstichprobe) gezogen und anhand der Daten $(<1 \%$ fehlende Werte pro Variable) eine exploratorische Maximum-Likelihood-Faktorenanalyse (ML-Faktorenanalyse; EFA) mit der Software Mplus Version 8 (Muthén \& Muthén, 2017) mit obliquer Geomin-Rotation berechnet. Um über die Anzahl der zu extrahierenden latenten Faktoren zu entscheiden, wurde eine Parallelanalyse nach Horn (1965) durchgeführt. Dabei wird der empirisch gefundene Eigenwertverlauf mit einem zufällig ermittelten Eigenwertverlauf verglichen. Der Schnittpunkt der Verläufe indiziert die Anzahl zu extrahierender Faktoren.

Zur Evaluation der Anpassungsgüte des resultierenden Modells wird in allen Teilstudien der vorliegenden Arbeit die $\chi^{2}$-Statistik, der Comparative Fit Index (CFI), der Tucker-Lewis Index (TLI), der Root Mean Square Error of Approximation (RMSEA) und das Standardized Root Mean Square Residual (SRMR) herangezogen. Der $\chi^{2}$-Statistik sollte aufgrund ihrer Anfälligkeit gegenüber großer Stichproben jedoch keine allzu gewichtige Bedeutung bei der Beurteilung der Modellgüte zugemessen werden. Eine angemessene Modellanpassung an die Daten wird allgemeinhin angenommen, wenn die Werte des CFI und $\mathrm{TLI} \geq .90$ sowie des RMSEA $\leq .08$ und $\mathrm{SRMR} \leq .10$ sind (z. B. Marsh, 2007).

Ein weiteres Ziel dieser Studie war es, die gefundene Faktorenstruktur anhand einer größeren Substichprobe von $N=1594$ Auszubildenden (ca. 67\% der Gesamtstichprobe) zu replizieren und zu prüfen, ob die Struktur unabhängig von Geschlecht und Ausbildungsgang invariant ist, das Messmodell also unabhängig von diesen Variablen gilt. Diese Frage der Generalisierbarkeit eines Messmodells über verschiedene Subgruppen ist ein wichtiger Aspekt der Konstruktvalidität von Messinstrumenten. Falls die SMS-5 in verschiedenen Gruppen unterschiedlich funktioniert, wären gefundene Unterschiede zwischen Subgruppen möglicherweise nur ein Artefakt unterschiedlich gut geeigneter Items, da sich die Bedeutung der latenten Variablen zwischen Subgruppen dann unterscheiden kann (Eid, Gollwitzer \& Schmitt, 2015), so dass Mittelwertvergleiche und Zusammenhangsanalysen $\mathrm{zu}$ verzerrten Ergebnissen führen können (Guenole \& Brown, 2014).

Für die Untersuchung wurden konfirmatorische Faktorenanalysen (KFA) berechnet. Dabei wurde der latente Faktor jeweils mittels Kodierung von Effekten identifiziert, indem der Durchschnitt aller Ladungen auf den Wert eins und der Durchschnitt aller Intercepts auf den Wert Null fixiert wurde (Little, Slegers \& Card, 2006). Die Invarianz der SMS-5 wurde anhand von vier genesteten Modellen geprüft. Diese Modelle umfassen die konfigurale (keine Invarianz von Parametern), schwache (Faktorladungen), starke (Faktorladungen + Item-Intercepts) und strikte Invarianz (Faktorladungen + Item-Intercepts + Item-Residualvarianzen). Liegen diese Formen der Invarianz vor, gelten Messinstrumente als vergleichbar in verschiedenen Gruppen, wenngleich das Vorliegen partieller Invarianz, d.h. Invarianz ist nur für einen Teil der Parameter gegeben, als hinreichend gilt (Byrne, Shavelson \& Muthén, 1989). Dabei muss beachtet werden, dass die strikte Invarianz, als höchstes Level der Messinvarianz, in der Praxis selten gestützt wird (Brown, 2006; Little, Preacher, Selig \& Card, 2007). Um über das Vorliegen von Invarianz zu entscheiden, sind Richtwerte für den Vergleich von genesteten Modellen bedeutsam. Dieser Vergleich kann mittels eines $\chi^{2}$-Differenztests durchgeführt werden, der allerdings den gleichen Problemen unterliegt wie der $\chi^{2}$-Test. Entsprechend haben sich Leitlinien für den Vergleich anderer Fit-Statistiken (z. B. CFI, RMSEA) etabliert. Änderungen im CFI $\geq-.01$, die mit einer Änderung des RMSEA von $\geq .015$ einhergehen, sprechen gegen das Vorliegen von Invarianz (Chen, 2007; Cheung \& Rensvold, 2002). Zudem gilt, dass die Fit-Indizes von TLI und RMSEA sparsame gegenüber weniger sparsamen Modellen bevorzugen (z. B. Marsh, 2007). Da in unserer Stichprobe die Verteilung von Geschlecht und Ausbildungsgang konfundiert ist, haben wir in der Invarianztestung über die Geschlechter Auszubildende in GTB (96\% männlich) und bei der Invarianztestung über Ausbildungsgänge die Frauen ( $4 \%$ in GTB) nicht berücksichtigt.

\section{Ergebnisse und Diskussion}

Faktorielle Struktur der SMS-5. Anhand der ML-Faktorenanalyse zeigte sich eine akzeptable Passung des Ein-Faktor-Modells auf die Daten mit $\chi^{2}(5, N=801)=39.952$, $p<.001, \mathrm{RMSEA}=.093, \mathrm{CFI}=.956, \mathrm{TLI}=.911$ und SRMR $=.031$. Der Vergleich empirischer $(2.45 ; 0.82 ; 0.72 ; 0.57$; $0.45)$ und zufälliger $(1.10 ; 1.04 ; 1.00 ; 0.96 ; 0.90)$ Eigen- 
Tabelle 1. Prompt, Items und Kennwerte der SMS-5 in Studie 1 (Substichprobe 2)

Prompt. Beantworten Sie bitte spontan die folgenden Aussagen zu Ihrem momentanen Befinden.

Kreuzen Sie dazu bitte in jeder Zeile ein Kästchen unter den Zahlen zwischen 1 = „völlig unzutreffend“ und 7 = "trifft ganz genau zu“ an, um auszudrücken, wie sehr die jeweilige Aussage im Moment auf Sie zutrifft.

(Please reply spontaneously to the following statements about how you feel at the moment. Please tick one box between 1 'not true' and 7 'very true' in each line to express which statement applies to you at the moment.)

\begin{tabular}{|c|c|c|c|c|c|}
\hline \multirow{2}{*}{ Item } & & \multicolumn{4}{|c|}{ SMS-5 $(N=1594 ; \alpha=.73)$} \\
\hline & & $M$ & $S D$ & $r_{i t}$ & $\mathrm{FL}$ \\
\hline $01 *$ & $\begin{array}{l}\text { Ich fühle mich erledigt. } \\
\text { (I feel drained.) }\end{array}$ & 5.42 & 1.64 & .53 & .58 \\
\hline 02 & $\begin{array}{l}\text { Ich fühle mich ausgeglichen. } \\
\text { (I feel calm and rational.) }\end{array}$ & 4.26 & 1.55 & .39 & .50 \\
\hline $03 *$ & $\begin{array}{l}\text { Ich fühle mich antriebslos. } \\
\text { (I feel lazy.) }\end{array}$ & 5.46 & 1.55 & .55 & .51 \\
\hline 04 & $\begin{array}{l}\text { Ich fühle mich wach und konzentriert. } \\
\text { (I feel sharp and focused.) }\end{array}$ & 4.13 & 1.64 & .53 & .74 \\
\hline $05^{*}$ & $\begin{array}{l}\text { Ich fühle mich als hätte ich keine Willenskraft mehr. } \\
\text { (I feel like my willpower is gone.) }\end{array}$ & 6.25 & 1.27 & .48 & .42 \\
\hline SMS-5 & & 5.11 & 1.07 & & \\
\hline
\end{tabular}

Anmerkungen: SMS-5 = 5-Item-Skala zur Messung der momentan verfügbaren Selbstkontrollkapazität; $r_{\text {it }}=$ part-whole korrigierte Item-Trennschärfen; $\mathrm{FL}=$ Standardisierte Faktorladungen; ${ }^{*}=$ Rekodierte Items, Alle Faktorladungen sind statistisch signifikant $(p<.001)$.

wertverläufe spricht ebenfalls für die Extraktion eines einzelnen Faktors. Die Ladungen der 5 Items auf dem theoretisch zu erwartenden latenten Faktor waren signifikant und lagen zwischen $.41 \leq \lambda \leq .73$.

In einem nächsten Schritt sollte die Replizierbarkeit der eindimensionalen Struktur der SMS-5 anhand einer größeren Substichprobe untersucht werden, wobei einfaktorielle konfirmatorische Faktorenmodelle spezifiziert wurden. Bei der Betrachtung der Fit-Statistiken $\chi^{2}(5, N=$ $1594)=147.124, p<.001$, RMSEA $=.134$, CFI $=.910$, $\mathrm{TLI}=.821, \mathrm{SRMR}=.044$ zeigten sich unzureichende Werte für den RMSEA und TLI, weshalb wir aufgrund einer möglichen methodenbedingten Messfehlervarianz (Podsakoff, MacKenzie, Lee \& Podsakoff, 2003) die Residualkorrelationen zwischen negativ formulierten Items (1, 3 und 5; vgl. Tabelle 1) bei der eindimensionalen Modellspezifikation zuließen. Da die Daten anhand des Modells nun hinreichend gut beschrieben wurden $\chi^{2}(2, N=$ $1594)=26.436, p<.001$, RMSEA $=.088, \mathrm{CFI}=.985$, $\mathrm{TLI}=.923$, SRMR $=.017$ und alle Items signifikant $(p<.001)$ und substanziell $(\geq .42)$ auf dem latenten Faktor luden (vgl. Tabelle 1), konnte die postulierte eindimensionale interne Struktur der SMS-5 basierend auf den Befunden von EFA und KFA angenommen werden $(\alpha=.73)$. In allen nachfolgenden Studien wurde zur Evaluation der internen Struktur der SMS-5 die eindimensionale Modellspezifikation inklusive Residualkorrelationen analog zu Studie 1 übernommen. Dieses Vorgehen vereinfacht Vergleiche zwischen den internen Validitäten der $S M S-5$ in den unterschiedlichen Stichproben.

\section{Invarianz der SMS-5 über Personengruppen.}

Invarianz zwischen Männern und Frauen. Der Vergleich des Modells mit konfiguraler Invarianz mit einem Modell mit gleichgesetzten Ladungen unterstützte die Annahme schwacher Invarianz über Geschlechter, da das sparsamere Modell gemessen an den etablierten Richtlinien keine schlechtere Anpassung an die Daten lieferte, der RMSEA für das restriktivere Modell sogar besser war $(\Delta \mathrm{CFI}=-.004, \Delta \mathrm{RMSEA}=-.003)$. Der Vergleich dieses Modells mit einem Modell mit zusätzlich gleichgesetzten Item-Intercepts unterstützte die Annahme starker Invarianz $(\Delta \mathrm{CFI}=-.006, \Delta \mathrm{RMSEA}=.002)$. Auch die Annahme strikter Messinvarianz wurde mit einer Verbesserung im RMSEA gestützt $(\triangle \mathrm{CFI}=-.003, \Delta \mathrm{RMSEA}=-.005)$.

Invarianz zwischen GTB, IK und LAB. Der Test auf Messinvarianz zwischen den Ausbildungsgängen stützte die Annahme schwacher Invarianz durch einen besseren Wert im RMSEA für das restriktivere Modell $(\triangle \mathrm{CFI}=$ -.002, $\triangle$ RMSEA = -.024). Für die Annahme starker Invarianz zeigte sich jedoch keine Evidenz $(\triangle \mathrm{CFI}=-.044$, $\triangle$ RMSEA $=.033$ ), so dass wir sukzessive Item-Intercepts frei zwischen den Gruppen variieren ließen, um partielle starke Invarianz zu testen. Ein Modell, in dem wir die Intercepts der Items 1, 2 und 3 frei variieren ließen, stützte die Annahme partieller starker Invarianz, wiederum mit einem verbesserten RMSEA $(\Delta \mathrm{CFI}=-.002, \triangle \mathrm{RMSEA}=$ -.003). In dem resultierenden Modell waren folglich noch zwei Intercepts zwischen den Gruppen gleichgesetzt, was der Mindestanforderung an partielle Invarianz entspricht (Steenkamp \& Baumgartner, 1998). Für die Annahme 
strikter bzw. partieller strikter Invarianz fanden wir keine Unterstützung, wobei die Differenzen im RMSEA teilweise noch im Rahmen der oben berichteten Grenzwerte lagen $(\Delta$ CFI $\geq-.024, \Delta$ RMSEA $\geq$.013).

\section{Studie 2}

Ziel der zweiten Validierungsstudie war es, interne und externe Validitätsindikatoren der SMS-5, der 10-Item-Skala SSCCS- $K$ - $D$ und der 25-Item-Skala SSCCS- $D$ vergleichend zu evaluieren. Da sich die momentan verfügbare Selbstkontrollkapazität prädiktiv für Leistungen in unmittelbar nachfolgenden kognitiven Fähigkeitstests (z. B. Schmeichel et al., 2003) und Konzentrationstests (Friese et al., 2012) erweisen sollte, erwarteten wir substanziell positive $\mathrm{Zu}$ sammenhänge zwischen der SMS-5 und Leistungen in nachfolgenden kognitiven Fähigkeits- sowie Konzentrationstests. Personen mit einer hohen momentan verfügbaren Selbstkontrollkapazität sollten demnach besser in der Lage sein, irrelevante Reize zu unterdrücken und ihre Aufmerksamkeit auf die Testaufgaben zu fokussieren, wodurch bessere Testleistungen erzielt werden sollten. Da die Selbstkontrollkapazität situativen Schwankungen unterliegt und demnach nur mit zeitlich nahen kognitiven Leistungen in Verbindung stehen sollte, erwarteten wir jedoch keine signifikanten Zusammenhänge mit distalen Leistungsmaßen wie dem Notendurchschnitt und Leistungen in einem anderntags durchgeführten $\mathrm{Ma}-$ thematiktest. In den Validierungsstudien von Ciarocco et al. (2007) und Bertrams et al. (2011) zeigten sich zudem positive Zusammenhänge zwischen der dispositionellen und der momentanen Selbstkontrollkapazität und positive Korrelationen mit sozial erwünschtem Antwortverhalten. Dies ist dadurch zu erklären, dass sowohl sozial erwünschtes als auch selbstkontrolliertes Verhalten für eine gute soziale Anpassung und psychische Gesundheit kennzeichnend sind (Musch, Brockhaus \& Bröder, 2002). Korrelationen mit externen Validierungskriterien erwiesen sich aber bei Bertrams et al. (2011) auch nach Kontrolle sozialer Erwünschtheit als robust. Insgesamt erwarteten wir vergleichbare Befundmuster hinsichtlich der externen Indikatoren, auch unter Kontrolle sozial erwünschter Antworttendenzen der Teilnehmenden. Da die Items der SMS-5 vorrangig beanspruchen die momentan verfügbare Selbstkontrollkapazität im Lern- und Leistungskontext abzubilden, sollte die Skala trotz ihrer Kürze den längeren Skalenversionen (SSCCS-K-D, SSCCS- $D$ ) in der Vorhersage externer Leistungskriterien zumindest nicht unterlegen sein.

\section{Methode}

Stichprobe und Vorgehen. Untersucht wurden $N=129$ Schülerinnen und Schüler der zehnten Klasse aus sechs Gemeinschaftsschulen in Schleswig-Holstein (49\% weiblich; $\left.M_{\text {Alter }}=15.6, S D_{\text {Alter }}=0.70\right)$. Sie bearbeiteten zu Beginn der Untersuchung die 25 Items der SSCCS-D und im Anschluss zwei kognitive Leistungstests sowie weitere Fragebögen. Drei Wochen nach der ersten Testung bearbeiteten die Schülerinnen und Schüler einen Mathematiktest.

Messinstrumente. Die Instruktionen und das Antwortformat der SSCCS-D, SSCCS-K-D und SMS-5 (siehe Tabelle 2) wurden analog zu Studie 1 eingesetzt. Die kognitiven Fähigkeiten wurden über die Summenscores der Subtests Wortaufgaben, Zahlenreihen und Figurenanalogien des Kognitiven Fähigkeitstests für 4. bis 12. Klassen, Revision erfasst (KFT 4-12+R; Heller \& Perleth, 2000). Die Selbstkontrollleistung der Teilnehmenden wurde anhand der Fehlerzahl im revidierten d2-Konzentrationstest (d2-R; Brickenkamp, Schmidt-Atzert \& Liepmann, 2010) gemessen. Für die erfolgreiche Bewältigung des d2-R ist Selbstkontrolle notwendig (Friese et al., 2012), um dem Impuls zu widerstehen visuell ähnliche Distraktoren durchzustreichen und stattdessen lediglich die Zielzeichen fehlerfrei zu markieren. Als Fehler gelten ausgelassene Zielzeichen und durchgestrichene Distraktoren. Die dispositionelle Selbstkontrolle wurde mit der Deutschen Kurzversion (SCS-K-D; Bertrams \& Dickhäuser, 2009; Lindner, Nagy \& Retelsdorf, 2015) der Self-Control Scale von Tangney, Baumeister und Boone (2004) erfasst. Die Skala besteht aus 13 Aussagen (z. B. „Ich wünschte ich hätte mehr Selbstdisziplin.") mit einer fünfstufigen Likert-Skala. Zudem wurde die Soziale-Erwünschtheitsskala-17 (SES-17; Stöber, 1999) eingesetzt (z. B. „Ich lästere gelegentlich über andere hinter deren Rücken."). Die Schülerinnen und Schüler wurden gebeten den Notendurchschnitt ihres letzten Zeugnisses anzugeben. An einem separaten Testtag bearbeiteten sie zudem 23 Aufgaben zum mathematischen Problemlösen (Katzenbach et al., 2009). Interne Konsistenzen der eingesetzten Instrumente finden sich in Tabelle 3 .

Analysen. Neben einer erneuten Prüfung der internen Struktur der SMS-5 haben wir in dieser Studie die Mittelwerte, Standardabweichungen, part-whole korrigierte Trennschärfen und standardisierte Faktorladungen der einzelnen Items für die SMS-5 sowie SSCCS-K-D und SSCCS-D berechnet und vergleichend gegenübergestellt. Zur Untersuchung der konvergenten Validität der SMS-5 wurden Korrelationen zwischen der Skala und dem Summenscore im KFT und der Fehleranzahl im d2 sowie weitere konvergente und divergente externe Kriterien betrachtet. Da die Messgenauigkeit immer von der Anzahl 
der Items pro Skala abhängt (Spearman, 1904), untersuchten wir die minderungskorrigierten Korrelationen der Skalen mit den externen Kriterien. Anhand der einfachen Attenuitätskorrektur konnte geschätzt werden, wie hoch die Korrelation mit externen Kriterien ausfallen würden, wenn die momentan verfügbare Selbstkontrollkapazität jeweils messfehlerfrei erfasst würde, was einen aussagekräftigeren Vergleich der Korrelationen der drei Skalenvarianten mit den externen Kriterien erlaubt.

\section{Ergebnisse und Diskussion}

Faktorielle Struktur der SMS-5. Um die interne Struktur der SMS-5 in einer weiteren Stichprobe zu untersuchen, haben wir analog zu Studie 1 eine konfirmatorische Faktorenanalyse durchgeführt. Der Fit erwies sich erneut als sehr gut, $\chi^{2}(2, N=129)=3.360, p=.18$, RMSEA $=.073$, $\mathrm{CFI}=.991, \mathrm{TLI}=.957$ und SRMR $=.021$.

Item- und Skalenkennwerte im Vergleich. Tabelle 2 zeigt die Mittelwerte, Standardabweichungen, part-whole korrigierte Trennschärfen und standardisierte Faktorladungen der einzelnen Items der SMS-5, SSCCS- $K-D$ und SSCCS-D. Mit Ausnahme von den Items 8 und 11 zeigten sich ausreichend hohe bis sehr hohe Trennschärfen der jeweils zu den drei Skalen zugeordneten Items. Die interne Konsistenz der drei Skalenvarianten veränderte sich nach Ausschluss der beiden Items jedoch nicht, weshalb diese beibehalten wurden. Alle Items luden je nach $\mathrm{Zu}$ ordnung zu der SMS-5, SSCCS-K-D und SSCCS-D mindestens hinreichend hoch $(\geq .34)$ auf dem jeweils eindimensionalen latenten Faktor. Die interne Konsistenz der drei Skalenvarianten lag zwischen $.75 \leq \alpha \leq .90$ und ist somit jeweils mindestens akzeptabel.

Externe Validität der SMS-5. Wie Tabelle 3 darstellt, zeigten Personen mit einer hohen momentan verfügbaren Selbstkontrollkapazität bessere Leistungen im KFT und machten weniger Fehler bei der Inhibitionsaufgabe im d2. Das Korrelationsmuster erwies sich als relativ stabil über alle drei Skalenversionen sowie unter Kontrolle sozial erwünschter Antworttendenzen. Anzumerken ist, dass in Bezug auf proximale Leistungsmaße die Vorhersagekraft der ökonomischen SMS-5 der 25-Item-SSCCS-D-Version nicht unterlegen und der SSCCS-K-D (10-Items) sogar tendenziell überlegen war. Dies zeigt die Betrachtung minderungskorrigierter Korrelationen $\left(r_{\min }\right)$. Die Varianzaufklärung bei der Vorhersage der konvergenten Leistungskriterien fiel für die SMS-5 in Relation zur SSCCS-K-D sogar marginal höher aus $\left(\Delta R^{2}=.02\right)$; in Relation zur SSCCS-D ergaben sich keine Unterschiede $\left(\Delta R^{2}=.00\right)$. Erwartungsgemäß zeigten sich keine signifikanten Zusammenhänge zwischen den drei Skalen und dem Notendurchschnitt und der Mathematikleistung an einem anderen
Testtag, sowie dem Alter und Geschlecht der Teilnehmenden.

Analog zu Bertrams et al. (2011) fanden wir positive $\mathrm{Zu}-$ sammenhänge der SMS-5, SSCCS-K-D und SSCCS-D mit dispositioneller Selbstkontrolle und sozialer Erwünschtheit. Allerdings fallen diese Korrelationen für die SMS-5 gegenüber der SSCCS-K-D und SSCCS-D geringer aus. Dies weist darauf hin, dass die für den Leistungskontext selegierten $S M S$-5-Items die momentan verfügbare Selbstkontrollkapazität spezifischer abbilden als die breiter gefassten SSCCS-K-D- und SSCCS-D-Skalen. Daraus resultiert möglicherweise auch die geringere Korrelation der SMS-5 mit der breiter konzeptualisierten Trait-Skala zur Messung der dispositionellen Selbstkontrolle. Darüber hinaus scheint die $S M S-5$ im Vergleich zu den längeren Skalenversionen allerdings tendenziell robuster gegenüber sozial erwünschtem Antwortverhalten zu sein. Insgesamt zeigten sich hohe Korrelationen zwischen der $S M S-5$, der SSCCS- $K-D$ und SSCCS-D, die nach der Minderungskorrektur erwartungsgemäß noch größer ausfielen und selbst nach Kontrolle sozial erwünschter Antworttendenzen stabil blieben.

\section{Studie 3}

Ziel der dritten Studie war, korrelative Zusammenhänge zwischen der SMS-5 und affektiven Variablen sowie dem Stresserleben in Anlehnung an die Studien von Bertrams et al. (2011) und Ciarocco et al. (2007) zu untersuchen. Hierfür wurden Kriterien gewählt, die in identischer oder ähnlicher Form in den Validierungsstudien der Originalskalen herangezogen wurden, um die Vergleichbarkeit der Befunde zu gewährleisten.

Bei Bertrams et al. (2011) ergaben sich hohe positive Zusammenhänge zwischen der momentan verfügbaren Selbstkontrollkapazität und der aktuellen Stimmung sowie geringe negative Zusammenhänge mit emotionaler Erregtheit. Da sich eine positive Stimmung günstig auf die momentane Selbstkontrollkapazität auswirkt (Tice, Baumeister, Shmueli \& Muraven, 2007), haben wir angenommen, dass eine positive emotionale Bewertung des Untersuchungstages positiv mit der momentanen Selbstkontrollkapazität zusammenhängt. Da dieser keine besondere emotionale Ladung aufweisen sollte (d.h. ein gewöhnlicher Tag an der Universität), erwarteten wir keine bedeutsamen Zusammenhänge zwischen der momentan verfügbaren Selbstkontrollkapazität und des Erlebens emotionaler Erregtheit.

Des Weiteren erleben Studierende in Lernphasen häufig Stress und müssen Selbstkontrolle aufwenden, um Prüfungstermine einzuhalten (Ciarocco et al., 2007). Ge- 
Tabelle 2. Formulierungen der Items und Kennwerte der SSCCS-D, SSCCS-K-D und der SMS-5

\begin{tabular}{|c|c|c|c|c|c|c|c|c|c|}
\hline \multirow{2}{*}{$\overline{\text { Item }}$} & & \multirow[b]{2}{*}{ M } & \multirow[b]{2}{*}{$S D$} & \multicolumn{2}{|c|}{$\begin{array}{l}\text { SSCCS-D } \\
\alpha=.90\end{array}$} & \multicolumn{2}{|c|}{$\begin{array}{l}\text { SSCCS-K-D } \\
\quad \alpha=.81\end{array}$} & \multicolumn{2}{|c|}{$\begin{array}{l}\text { SMS-5 } \\
\alpha=.75\end{array}$} \\
\hline & & & & $r_{\text {it }}$ & $\mathrm{FL}$ & $r_{\text {it }}$ & $\mathrm{FL}$ & $r_{\text {it }}$ & $\mathrm{FL}$ \\
\hline $01 *$ & Ich fühle mich mental erschöpft. & 5.28 & 1.35 & .64 & .72 & & & & \\
\hline $02 *$ & $\begin{array}{l}\text { Es würde mir im Moment schwer fallen, mich auf etwas zu konzentrie- } \\
\text { ren. }\end{array}$ & 5.42 & 1.52 & .54 & .56 & & & & \\
\hline $03 *$ & Ich brauche etwas Angenehmes, um mich besser zu fühlen. & 4.60 & 1.94 & .46 & .51 & .47 & .52 & & \\
\hline 04 & Ich bin motiviert. & 4.91 & 1.53 & .40 & .39 & & & & \\
\hline $05^{\star}$ & $\begin{array}{l}\text { Wenn ich jetzt eine schwierige Aufgabe gestellt bekäme, würde } \\
\text { ich schnell aufgeben. }\end{array}$ & 5.58 & 1.48 & .49 & .48 & & & & \\
\hline $06 *$ & Ich fühle mich erledigt. & 5.30 & 1.54 & .64 & .71 & .63 & .69 & .61 & .67 \\
\hline 07 & Ich habe viel Energie. & 4.73 & 1.54 & .49 & .53 & & & & \\
\hline $08 *$ & Ich fühle mich abgespannt. & 4.82 & 1.28 & .22 & .27 & & & & \\
\hline $09 *$ & $\begin{array}{l}\text { Wenn ich im Augenblick durch etwas in Versuchung geführt würde, } \\
\text { würde es mir sehr schwer fallen, zu widerstehen. }\end{array}$ & 4.99 & 1.74 & .40 & .44 & .37 & .45 & & \\
\hline $10 *$ & $\begin{array}{l}\text { Ich würde jede schwierige Aufgabe, die mir gestellt würde, aufgeben } \\
\text { wollen. }\end{array}$ & 5.98 & 1.24 & .57 & .58 & .52 & .56 & & \\
\hline 11 & Ich fühle mich ausgeglichen. & 4.40 & 1.55 & .28 & .32 & .30 & .31 & .35 & .34 \\
\hline $12^{*}$ & Ich kann keine Information mehr aufnehmen. & 6.28 & 1.04 & .62 & .67 & .55 & .63 & & \\
\hline $13^{*}$ & Ich fühle mich antriebslos. & 5.68 & 1.25 & .75 & .80 & .75 & .84 & .64 & .83 \\
\hline $14^{\star}$ & $\begin{array}{l}\text { Im Moment würde es mir schwer fallen, Pläne für die Zukunft } \\
\text { zu schmieden. }\end{array}$ & 4.85 & 1.87 & .32 & .36 & & & & \\
\hline 15 & Ich fühle mich wach und konzentriert. & 4.48 & 1.59 & .60 & .62 & .51 & .55 & .52 & .57 \\
\hline $16^{*}$ & Ich möchte aufgeben. & 6.56 & 0.99 & .57 & .60 & .54 & .60 & & \\
\hline 17 & $\begin{array}{l}\text { Dies wäre ein guter Zeitpunkt für mich, um eine wichtige Entscheidung } \\
\text { zu treffen. }\end{array}$ & 3.23 & 1.61 & .33 & .33 & & & & \\
\hline $18 *$ & Ich fühle mich als hätte ich keine Willenskraft mehr. & 6.14 & 1.21 & .61 & .70 & .60 & .69 & .51 & .67 \\
\hline $19 *$ & $\begin{array}{l}\text { Es fällt mir schwer, momentan meine Aufmerksamkeit aufrecht- } \\
\text { zuerhalten. }\end{array}$ & 5.70 & 1.35 & .57 & .62 & & & & \\
\hline 20 & Ich bin im Moment in der Lage, mich gut konzentrieren zu können. & 5.34 & 1.46 & .59 & .59 & & & & \\
\hline 21 * & Mein geistiger Akku ist leer. & 5.88 & 1.32 & .66 & .73 & & & & \\
\hline 22 & Im Augenblick würde mich eine neue Herausforderung reizen. & 4.19 & 1.91 & .30 & .31 & & & & \\
\hline $23 *$ & Ich würde mich gerne eine Weile entspannen. & 3.71 & 1.93 & .61 & .65 & & & & \\
\hline $24^{*}$ & Es fällt mir gerade schwer, meine Bedürfnisse zu kontrollieren. & 5.73 & 1.36 & .35 & .40 & & & & \\
\hline $25^{\star}$ & Ich fühle mich entmutigt. & 6.22 & 1.09 & .54 & .58 & & & & \\
\hline SSCC & S-D & 5.20 & 0.80 & & & & & & \\
\hline SSCC & CS-K-D & 5.44 & 0.88 & & & & & & \\
\hline SMS & & 5.20 & 1.01 & & & & & & \\
\hline
\end{tabular}

Anmerkungen: SSCCS-D = State Self-Control Capacity Scale Deutsch; SSCCS-K-D = State Self-Control Capacity Scale Kurzversion Deutsch; SMS- 5 = 5 Item-Skala zur Messung der momentan verfügbaren Selbstkontrollkapazität; $r_{\text {it }}=$ part-whole korrigierte Item-Trennschärfen; FL = Standardisierte Faktorladungen; ${ }^{*}=$ rekodierte Items. Alle Faktorladungen sind statistisch signifikant $(p<.001)$.

mäß der Annahme von Inzlicht und Legault (2014) werden Personen durch das Erleben von Stress alarmiert, Ziel-irrelevante Reize zu regulieren oder zu unterdrücken, um ihre übergeordneten Ziele weiterhin zu verfolgen (Kotabe \& Hofmann, 2015). Ein hoher Ausprägungsgrad des erlebten Stresspegels sollte somit einen höheren Verbrauch der Selbstkontrollkapazität nach sich ziehen, wodurch sich ein negativer Zusammenhang zwischen dem aktuellen und dem retrospektiv eingeschätzten Stresspe- gel und der momentanen Selbstkontrollkapazität einstellen sollte. Wie von Bertrams et al. (2011) und Ciarocco et al. (2007) gezeigt, erwarteten auch wir negative Zusammenhänge zwischen der momentan verfügbaren Selbstkontrollkapazität und dem erlebten Gesamtstress am Untersuchungstag sowie in den unmittelbar zurückliegenden Tagen. Zudem sollte die Anzahl der in Lehrveranstaltungen verbrachten Stunden negativ mit der momentan verfügbaren Selbstkontrollkapazität korrelieren, da durch die 


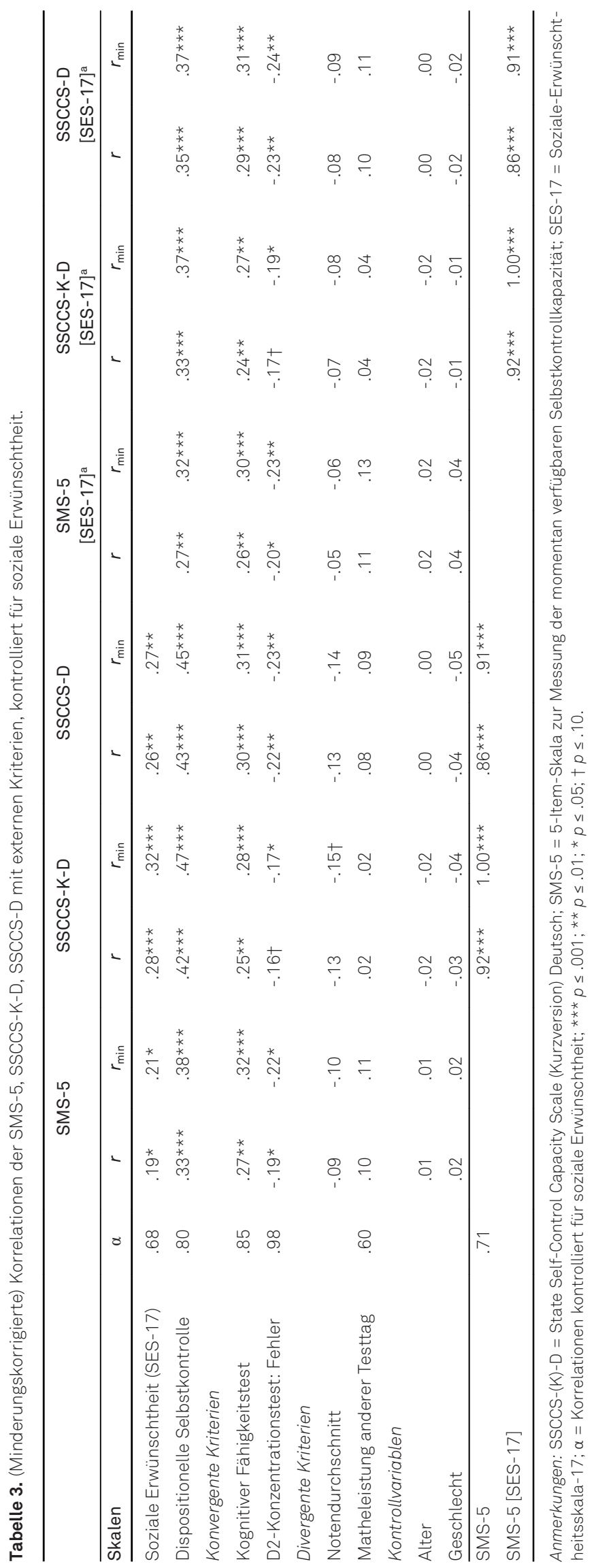


notwendige Aufmerksamkeitsfokussierung der Studierenden in den Lehrveranstaltungen kurzfristig ein hohes Maß an Selbstkontrollkapazität erschöpft wird (Bertrams et al., 2011).

\section{Methode}

Stichprobe und Vorgehen. Die SMS-5 und weitere Fragebögen wurden $N=95$ Lehramtsstudierenden im Bachelorstudium ( $58 \%$ weiblich; $M_{\text {Alter }}=20.64, S D_{\text {Alter }}=2.50$ ) während einer Vorlesung an der Christian-Albrechts-Universität Kiel vorgelegt.

Messinstrumente. Neben der Bearbeitung der SMS-5 gaben die Studierenden an, wie viele Stunden sie an dem Untersuchungstag bereits in Lehrveranstaltungen verbracht haben. Anhand einer adaptierten Version der Valenz- und Erregungs-Skala des Self-Assessment-Manikin (Bradley \& Lang, 1994) sollten sie zudem ihre bisherigen Tageserlebnisse jeweils auf einer neunstufigen Skala von $1=$,sehr unangenehm" bis $9=$,sehr angenehm" und von $1=$,gar nicht aufregend" bis $9=$, ,sehr aufregend" bewerten. Anschließend legten wir analog zu Bertrams et al. (2011) und Ciarocco et al. (2007) jeweils ein Item vor, auf dem der erlebte Stress am Untersuchungstag sowie in den letzten sieben Tagen eingeschätzt werden sollte (von $1=$,niedrig" bis $7=$,hoch“).

Analysen. Die interne Struktur der SMS-5 wurde analog zu den Studien 1 und 2 untersucht. Zur Untersuchung der externen Validität der SMS-5 wurden Korrelationen und attenuitätskorrigierte Korrelationen (vgl. Studie 2) zwischen der Skala und den externen Kriterien berechnet.

\section{Ergebnisse und Diskussion}

Faktorielle Struktur der SMS-5. In einer konfirmatorischen Faktorenanalyse erwies sich der Modell-Fit der eindimensionalen $S M S-5$ erneut als sehr gut, $\chi^{2}(2, N=95)=3.219$, $p=.20, \mathrm{RMSEA}=.080, \mathrm{CFI}=.984, \mathrm{TLI}=.921$ und SRMR $=$ .029, was die Robustheit der internen Struktur der Skala unterstreicht. Die interne Konsistenz der SMS-5 lag in dieser Stichprobe bei $\alpha=.71$ und war somit akzeptabel.

Externe Validität der SMS-5. Wie schon in Studie 2 ergaben sich auch hier erwartungskonform keine signifikanten Zusammenhänge zwischen der SMS-5 und dem Alter oder Geschlecht der Teilnehmenden. Es zeigte sich jedoch der angenommene signifikant positive Zusammenhang mit der emotionalen Bewertung der Erlebnisse am Untersuchungstag $\left(r=.55 ; r_{\min }=.65\right.$; jeweils $\left.p<.001\right)$. Dies unterstreicht die Ergebnisse von Tice et al. (2007), Bertrams et al. (2011) und Ciarocco et al. (2007), dass sich ein positiver affektiver Zustand günstig auf die momentane
Selbstkontrollkapazität auswirkt. Erwartungsgemäß zeigte sich kein signifikanter Zusammenhang der SMS-5 mit dem Erleben emotionaler Erregtheit am Untersuchungstag.

Wie bei Bertrams et al. (2011) und Ciarocco et al. (2007) zeigten sich signifikant negative Zusammenhänge zwischen der momentan verfügbaren Selbstkontrollkapazität und dem Stresserleben am Untersuchungstag $(r=$ $-.37 ; r_{\min }=-.44$; jeweils $\left.p<.001\right)$ und in der vorangegangenen Woche $\left(r=-.24 ; r_{\min }=-.28\right.$; jeweils $\left.p<.05\right)$. Ebenfalls stand die Anzahl der verbrachten Stunden in Lehrveranstaltungen am Untersuchungstag wie von Bertrams et al. (2011) postuliert, in einem negativen Zusammenhang mit der momentan verfügbaren Selbstkontrollkapazität $\left(r=-.23 ; r_{\min }=-.28\right.$; jeweils $\left.p<.05\right)$. Insgesamt wiesen die Befunde auf eine Vergleichbarkeit der prädiktiven Validität der SSCCS-D mit der SMS-5 hin.

\section{Studie 4}

Ziel der vierten Teilstudie war es, die Änderungssensitivität der SMS-5 zu untersuchen. Die Ausübung von Selbstkontrolle führt im zeitlichen Verlauf zu einer Reduktion der Selbstkontrollleistung (Lindner et al., 2017). Baumeister et al. (2007) postulieren, dass dieses Phänomen durch eine kurzfristige Reduktion in der individuell verfügbaren Selbstkontrollkapazität erklärt werden kann. Bertrams et al. (2011) zeigen, dass Veränderungen der momentan verfügbaren Selbstkontrollkapazität mit der SSCCS-D sensitiv gemessen werden können. Ebenso sollte auch die SMS-5 Veränderungen der momentan verfügbaren Selbstkontrollkapazität im zeitlichen Verlauf abbilden können, wenn beispielsweise Studierende bei der Bearbeitung von Lern- und Testaufgaben ihre Aufmerksamkeit und Gedanken über längere Zeit auf die Aufgabeninhalte fokussieren müssen, wodurch die momentane Selbstkontrollkapazität im zeitlichen Verlauf abnehmen sollte (Lindner et al., 2018). Personen mit einer stärkeren Abnahme der momentanen Selbstkontrollkapazität sollten die Aufgabenbearbeitungen zudem als anstrengender erleben als Personen mit einer geringeren Abnahme. Um sicherzustellen, dass die postulierten Veränderungen in der momentanen Selbstkontrollkapazität mit der SMS-5 valide messbar sind, untersuchen wir zudem die zeitliche Invarianz des Messmodells bei wiederholtem Einsatz der Skala. Aus messtheoretischer Sicht würde eine zeitliche Invarianz in Analogie zur oben beschriebenen Invarianz zwischen Gruppen bedeuten, dass die beobachtete momentane Selbstkontrollkapazität zu verschiedenen, kurz hintereinander folgenden Messzeitpunkten dasselbe variable Konstrukt erfasst (Eid et al., 2015). Die Bedeutung der latenten Variable sollte sich im zeitlichen Verlauf nicht ändern. 


\section{Methode}

Stichprobe und Vorgehen. Die SMS-5 wurde im Rahmen einer Lernstudie im Labor mit $N=140$ Studierenden $(88 \%$ weiblich; $\left.M_{\text {Alter }}=24.35, S D_{\text {Alter }}=3.84\right)$ unterschiedlicher Fächer der Christian-Albrechts-Universität Kiel wiederholt in Zeitabständen von jeweils ca. 20 bis 30 Minuten (1) zu Untersuchungsbeginn, (2) nach einer Lernphase zum Thema Mitose und Meiose, (3) nach zwei Untertests des KFT (N2 und N3; Heller \& Perleth, 2000) sowie (4) nach einem Leistungstest, bezogen auf die vorherige Lernphase, eingesetzt. Die momentan verfügbare Selbstkontrollkapazität wurde also insgesamt viermal in vergleichbaren Zeitabständen mit der SMS-5 $(.76 \leq \alpha \leq .85)$ gemessen. Zum Abschluss der Studie gaben die Studierenden an, wie anstrengend sie die zuvor bearbeiteten Aufgaben empfunden haben.

Messinstrumente. Neben der wiederholten Vorgabe der SMS-5 wurde die empfundene Anstrengung bei der Bearbeitung der Testaufgaben anhand von vier selbstkonstruierten Items (z. B. „Ich fand die Bearbeitung der Testaufgaben sehr anstrengend.") erfasst. Die Aussagen wurden auf einer Likert-Skala von $1=$,stimmt gar nicht" bis $4=$ „stimmt genau“ eingeschätzt $(\alpha=.85)$.

Analysen. Für die Untersuchung der Veränderungssensitivität der SMS-5 spezifizierten wir in Mplus 8 (Muthén \& Muthén, 2017) mehrere Curve-of-Factor-Modelle (McArdle, 1988) und latente Wachstumskurvenmodelle, bei denen nicht nur der mittlere Ausgangswert und die mittlere Veränderung, sondern auch die Indikatoren pro Messzeitpunkt als latente Variablen spezifiziert werden (Wachstumskurvenmodell zweiter Ordnung). Die Trajektorie der momentan verfügbaren Selbstkontrollkapazität wurde im zeitlichen Verlauf der Aufgabenbearbeitung anhand eines mittleren Basisniveaus (Intercept) und der mittleren Veränderung (Slope) über die vier Messzeitpunkte beschrieben. Pro Messzeitpunkt wurde die Ladung des latenten SMS-5-Faktors auf den Intercept-Faktor auf 1 fixiert. Zur Spezifikation des latenten Slope-Faktors wurde ein Modell mit linearem Veränderungstrend mit einem Modell mit frei geschätzter Veränderungsform verglichen.

Bei der Spezifikation von Curve-of-Factor-Modellen muss zunächst die Messinvarianz der Messmodelle der latenten Variablen über alle Messzeitpunkte evaluiert werden. Diese längsschnittliche Messinvarianz ist ebenso wie die in Studie 1 geprüfte Invarianz zwischen Gruppen ein wichtiges Validitätskriterium. Die Messinvarianz der $S M S-5$ über die Zeit wurde entsprechend der in Studie 1 beschriebenen Stufen der Invarianz getestet. Dabei wurden zusätzlich zu den beschriebenen Residualkorrelationen zwischen negativ formulierten SMS-5-Items auch
Korrelationen zwischen den Residuen identischer SMS-5Items über die Zeit zugelassen (Marsh \& Hau, 1996).

\section{Ergebnisse und Diskussion}

Messinvarianz der SMS-5 über die Zeit. Für die Prüfung der Messinvarianz über die Zeit wurde zunächst ein Modell mit konfiguraler Invarianz getestet, das mit $\chi^{2}(122, N=140)=$ $170.255, p<.01$, RMSEA $=.053$, CFI $=.977$, TLI $=.964$ und SRMR $=.055$ gut zu den Daten passte. Die Güte der Modellanpassung im restriktiveren Modell mit gleichgesetzten Faktorladungen über die Zeit war im CFI unverändert gut $(\triangle \mathrm{CFI}=.000)$, beim RMSEA sogar leicht verbessert ( $\triangle$ RMSEA $=-.003)$, so dass die Annahme schwacher Invarianz gestützt wurde. Beim Vergleich dieses Modells mit einem Modell mit starker Invarianz zeigte sich, dass nur die Differenz im RMSEA für die Invarianzannahme spricht $(\triangle \mathrm{RMSEA}=.012)$, während die Differenz im CFI das Kriterium überschreitet $(\Delta \mathrm{CFI}=-.015)$. Daher haben wir in einem weiteren Schritt partielle starke Invarianz getestet, indem die Intercepts der Items 1 und 3 frei über die Zeit variieren durften. Dieses Modell passte wiederum nicht wesentlich schlechter als das Modell mit schwacher Invarianz $(\Delta \mathrm{CFI}=-.009, \Delta \mathrm{RMSEA}=.007)$. Schließlich wurde durch den Vergleich dieses Modells mit einem Modell, in dem zusätzlich die Residualvarianzen für die Items mit invarianten Item-Intercepts gleichgesetzt wurden, auch die Annahme partieller strikter Invarianz gestützt $(\Delta \mathrm{CFI}=-.004, \Delta \mathrm{RMSEA}=.002)$.

Veränderungssensitivität der SMS-5. Das Wachstumskurvenmodell mit frei geschätzter Veränderung zeigte gegenüber dem restriktiveren Modell mit linearer Veränderungsform keine schlechtere Modellanpassung: $\Delta \chi^{2}(2)=2.35$, $p=.310$. Das resultierende Modell mit linearer Veränderungsform zeigte eine gute Modellanpassung: $\chi^{2}(157$, $N=140)=241.148, p<.001, \mathrm{RMSEA}=.062, \mathrm{CFI}=.959$, $\mathrm{TLI}=.951, \mathrm{SRMR}=.105$. Die mittlere momentan verfügbare Selbstkontrollkapazität (Intercept) der Studierenden lag zu Beginn der Untersuchung bei $M=4.96$ ( $S E=0.09$; $p<.001)$ und nahm im Verlauf der Untersuchung im Mittel um $M=-.82$ (SE = 0.10; $p<.001)$ ab (Slope). Diese latente Abnahme in der Selbstkontrollkapazität weist auf die postulierte Veränderungssensitivität der SMS-5 hin. Wie angenommen gaben Personen mit einem hohen Ausgangswert ihrer momentan verfügbaren Selbstkontrollkapazität an, dass sie die Aufgabenbearbeitung als weniger stark anstrengend empfanden als Personen mit niedriger Selbstkontrollkapazität $(r=-.26 ; p<.01)$. Ebenso bewerteten Studierende die zuvor bearbeiteten Testaufgaben als anstrengender $(r=-.50 ; p<.001)$, wenn ihre Selbstkontrollkapazität im zeitlichen Verlauf der Studienteilnahme stärker abnahm. 


\section{Gesamtdiskussion}

Das Ziel der vorliegenden Studie war, ein ökonomisches und valides 5-Item-Instrument zur Messung der momen$\tan$ verfügbaren Selbstkontrollkapazität im Lern- und Leistungskontext, basierend auf seligierten SSCCS- $K-D$ Items, zu entwickeln. Für die Untersuchung der internen und externen Validität der SMS-5 wurden anhand latenter Strukturgleichungsmodelle konfirmatorische Faktorenanalysen und (minderungskorrigierte) Korrelationen mit externen Kriterien berechnet. Die postulierte einfaktorielle Struktur der SMS-5 wurde in vier unabhängigen Stichproben weitestgehend gestützt. Für die SMS-5 zeigte sich, dass mindestens die Annahme partieller starker Messinvarianz zwischen Männern und Frauen, unterschiedlichen Ausbildungsgängen und über die Zeit angenommen werden kann. Teilweise wurden sogar höhere Niveaus der Messinvarianz gestützt. Die mit der SMS-5 gemessene momentan verfügbare Selbstkontrollkapazität stand zudem in einem positiven Zusammenhang mit den unmittelbaren Leistungen im kognitiven Fähigkeitstest sowie negativ mit den Fehlern im d2-Konzentrationstest. Keine Zusammenhänge zeigten sich zwischen der SMS-5 und divergenten Leistungskriterien (Notendurchschnitt und Mathematikleistung an einem anderen Testtag) sowie dem Alter und Geschlecht der Teilnehmenden in allen Teilstudien.

Die fünf Items der SMS-5 erwiesen sich in unseren Studien damit als geeignete Indikatoren, um die zentralsten Facetten selbstkontrollierten Verhaltens, nämlich die Inhibition irrelevanter Reize (vgl. Leistung im d2), sowie die Persistenz in kognitiven Fähigkeitstests (vgl. Leistung im KFT) ökonomisch vorherzusagen, ohne dabei der prädiktiven Validität der SSCCS-K-D oder SSCCS-D substanziell unterlegen zu sein.

Weiterhin zeigten sich signifikante positive Korrelationen mit der dispositionellen Selbstkontrolle, der emotionalen Bewertung des Untersuchungstages sowie dem Stresserleben. Die Zusammenhänge der SMS-5 mit dem zeitlich näherliegenden Stresserleben am Untersuchungstag fielen erwartungsgemäß größer aus als die Korrelation mit dem Stresserleben bezogen auf die vorangegangenen sieben Tage. Anhand latenter Wachstumskurvenmodelle konnte zudem gezeigt werden, dass die mit der $S M S$ 5 im Abstand von jeweils ca. 20-30 Minuten wiederholt gemessene momentan verfügbare Selbstkontrollkapazität von Studierenden vor und nach einer Lernphase sowie jeweils nach zwei Leistungstests im zeitlichen Verlauf signifikant abnahm und mit der empfundenen Anstrengung bei der Aufgabenbearbeitung in Verbindung stand.

Die Aussagekraft der vorliegenden Arbeit hat jedoch Grenzen. Einerseits erwies sich die interne Struktur der
SMS-5 in Studie 1 trotz der einbezogenen Residualkorrelationen in der eindimensionalen Modellspezifikation insgesamt zwar als akzeptabel, der RMSEA-Wert war jedoch nicht ideal. In zukünftigen Studien sollte deshalb die interne Validität der SMS-5 weiterhin evaluiert werden, wenngleich die Modellgüte in den Studien 2 bis 4 akzeptabel bis gut war. Eine Limitation liegt in der relativ kleinen Validierungsstichprobe und Ein-Item-Messungen externer Kriterien, die in Studie 3 für die Untersuchung von Zusammenhängen mit dem Stresserleben und affektiven Komponenten herangezogen wurden. Es wäre deshalb wünschenswert die SMS-5 in größeren und heterogeneren Stichproben sowie in emotional geladenen Kontexten erneut zu validieren um Zusammenhänge mit Skalen zur Messung affektiver Komponenten $\mathrm{zu}$ untersuchen und dabei auch Zusammenhänge mit den Big-Five Persönlichkeitsfaktoren zu berücksichtigen. Genauer wäre für eine weiterführende Validierung der $S M S-5$ von Interesse, inwieweit dispositionelle Anteile der momentan verfügbaren Selbstkontrollkapazität mit den Facetten von Neurotizismus, vor allem mit individuellen Ausprägungen der Impulskontrolle, in Verbindung stehen. Inwieweit sich die SMS-5 von Instrumenten zur Messung von kognitiver Erschöpfung oder Müdigkeit abgrenzt, ist ebenfalls eine Fragestellung, die es in zukünftigen Studien zu adressieren gilt. Notwendig wäre dabei auch die Untersuchung der Stabilität der Korrelationsmuster zwischen der SMS-5 und externen Kriterien, unter Kontrolle von Müdigkeitseffekten. Darüber hinaus wäre es wünschenswert die SMS-5 zur Vorhersage experimentell induzierter Ego-Depletion-Effekte einzusetzen. Durch dieses Vorgehen könnten Zusammenhänge zwischen der induzierten Selbstkontrollerschöpfung und der subjektiv empfundenen Verfügbarkeit der momentanen Selbstkontrollkapazität untersucht werden. Anzunehmen wäre, dass sich der experimentell induzierte Ego-Depletion-Effekt anhand der SMS-5, ähnlich wie mit der SSCCS in den Validierungsstudien von Bertrams et al. (2011) und Ciarocco et al. (2007) abbilden lässt. Die Befunde aus Studie 3 stützen diese Annahme, da die Leistung im d2-Konzentrationstest anhand der SMS-5 ebenso vorhergesagt wurde, wie in der Studie von Friese et al. (2012) durch eine experimentelle Ego-Depletion-Intervention. Die in der vorliegenden Studie gefundenen Zusammenhänge zwischen der SMS-5 und der Inhibitionsleistung sowie Persistenz in kognitiven Tests weisen bereits auf eine hohe Konstruktvalidität hin, was durch die änderungssensitive Abbildung der momentanen Selbstkontrollkapazität durch die $S M S-5$ im zeitlichen Verlauf untermauert wird. Darüber hinaus erwies sich die $S M S-5$ in einer aktuellen Studie von Lindner et al. (2018) ebenfalls als valides Instrument zur Messung von Veränderungen der momentan verfügbaren Selbstkontrollkapazität im Verlauf einer 140- 
minütigen mathematisch-naturwissenschaftlichen Leistungstestung. In zukünftigen Studien sollte jedoch untersucht werden, inwieweit ein kurzfristig wiederholter Einsatz der SMS-5 zu reaktivem Verhalten von Probandinnen und Probanden führen kann.

Nicht auszuschließen ist zudem, dass dem ökonomischen Vorteil der SMS-5 ein Verlust an prädiktivem Wert entgegensteht, wenn man die Skala zur Messung der momentan verfügbaren Selbstkontrollkapazität außerhalb des Lern- und Leistungsbereiches einsetzen möchte, um Zusammenhänge mit weiteren selbstkontrollassoziierten Kriterien zu betrachten, wie beispielsweise dem Widerstehen von Süßigkeiten während einer Diät. Das Ziel der vorliegenden Arbeit war jedoch eine Kurzskala zur ökonomischen und kurzfristig wiederholten Messung der situativen Selbstkontrollkapazität zu entwickeln, um Effekte selbstkontrollierten Verhaltens im Lern- und Leistungskontext (z. B. in Feldstudien) abzubilden. Insgesamt erwies sich die $S M S-5$ für dieses Einsatzfeld als valides, reliables und ökonomisches Instrument, um die momentan verfügbare Selbstkontrollkapazität und deren Veränderung zu messen und dadurch leistungsrelevante Outcome-Variablen vorherzusagen.

\section{Literatur}

Baumeister, R. F. \& Vohs, K. D. (2016). Misguided effort with elusive implications. Perspectives on Psychological Science, 11, 574-575. https://doi.org/10.1177/1745691616652878

Baumeister, R. F., Vohs, K. D. \& Tice, D. M. (2007). The strength model of self-control. Current Directions in Psychological Science, 16, 351-355. https://doi.org/10.1111/j.1467-8721.2007. 00534.x

Bertrams, A. \& Dickhäuser, O. (2009). Messung dispositioneller Selbstkontroll-Kapazität: Eine deutsche Adaptation der Kurzform der Self-Control Scale (SCS-K-D). Diagnostica, 55, 2-10. https://doi.org/10.1026/0012-1924.55.1.2

Bertrams, A., Englert, C. \& Dickhäuser, O. (2010). Self-control strength in the relation between trait test anxiety and state anxiety. Journal of Research in Personality, 44, 738-741. https://doi.org/10.1016/J.JRP.2010.09.005

Bertrams, A., Englert, C., Dickhäuser, O. \& Baumeister, R. F. (2013). Role of self-control strength in the relation between anxiety and cognitive performance. Emotion, 13, 668-680. https://doi.org/ $10.1037 / a 0031921$

Bertrams, A., Unger, A. \& Dickhäuser, O. (2011). Momentan verfügbare Selbstkontrollkraft - Vorstellung eines Messinstruments und erste Befunde aus pädagogisch-psychologischen Kontexten. Zeitschrift für Pädagogische Psychologie, 25, 185 196. https://doi.org/10.1024/1010-0652/a000042

Bradley, M. M. \& Lang, P. J. (1994). Measuring emotion: The selfassessment manikin and the semantic differential. Journal of Behavior Therapy and Experimental Psychiatry, 25, 49-59. Retrieved from http://www.ncbi.nlm.nih.gov/pubmed/7962581

Brickenkamp, R., Schmidt-Atzert, L. \& Liepmann, D. (2010). Test d2-Revision: Aufmerksamkeits- und Konzentrationstest. Göttingen: Hogrefe.
Brown, T. A. (2006). Confirmatory factor analysis for applied research. New York, NY: Guilford Press.

Byrne, B. M., Shavelson, R. J. \& Muthén, B. O. (1989). Testing for the equivalence of factor covariance and mean structures: The issue of partial measurement invariance. Psychological Bulletin, 105, 456-466. https://doi.org/10.1037/0033-2909.105.3.456

Chen, F. F. (2007). Sensitivity of goodness of fit indexes to lack of measurement invariance. Structural Equation Modeling: A Multidisciplinary Journal, 14, 464-504. https://doi.org/10. 1080/10705510701301834

Cheung, G. W. \& Rensvold, R. B. (2002). Evaluating goodness of fit indexes for testing measurement invariance. Structural Equation Modeling: A Multidisciplinary Journal, 9, 233 - 255. https:// doi.org/10.1207/S15328007SEM0902_5

Ciarocco, N. J., Twenge, J. M., Muraven, M. \& Tice, D. M. (2007). Measuring state self-control: Reliability, validity, and correlations with physical and psychological stress. Unpublished manuscript, Monmouth University, NJ, USA.

Clarkson, J. J., Hirt, E. R., Jia, L. \& Alexander, M. B. (2010). When perception is more than reality: The effects of perceived versus actual resource depletion on self-regulatory behavior. Journal of Personality and Social Psychology, 98, 29-46. https://doi. org/10.1037/a0017539

Diestel, S., Rivkin, W. \& Schmidt, K.-H. (2015). Sleep quality and self-control capacity as protective resources in the daily emotional labor process: Results from two diary studies. Journal of Applied Psychology, 100, 809-827. https://doi.org/10.1037/ a0038373

Eid, M., Gollwitzer, M. \& Schmitt, M. (2015). Statistik und Forschungsmethoden. Lehrbuch; mit Online-Materialien. Weinheim: Beltz.

Friese, M., Messner, C. \& Schaffner, Y. (2012). Mindfulness meditation counteracts self-control depletion. Consciousness and Cognition, 21, 1016-1022. https://doi.org/10.1016/j.concog.2012. 01.008

Germeys, L. \& De Gieter, S. (2018). A diary study on the role of psychological detachment in the spillover of self-control demands to employees' ego depletion and the crossover to their partner. European Journal of Work and Organizational Psychology, 27, 140 -152. https://doi.org/10.1080/1359432X.2017. 1417259

Guenole, N. \& Brown, A. (2014). The consequences of ignoring measurement invariance for path coefficients in structural equation models. Frontiers in Psychology, 5, 1-16. https://doi. org/10.3389/fpsyg.2014.00980

Hagger, M. S., Chatzisarantis, N. L. D., Alberts, H., Anggono, C. O., Batailler, C., Birt, A. R. et al. (2016). A multilab preregistered replication of the ego-depletion effect. Perspectives on Psychological Science, 11, 546-573. https://doi.org/10.1177/1745691 616652873

Hagger, M. S., Wood, C., Stiff, C. \& Chatzisarantis, N. L. D. (2010). Ego depletion and the strength model of self-control: A metaanalysis. Psychological Bulletin, 136, 495 - 525. https://doi.org/ $10.1037 / \mathrm{a} 0019486$

Heller, K. A. \& Perleth, C. (2000). Kognitiver Fähigkeitstest für 4. bis 12. Klassen, Revision. KFT 4-12+R. Göttingen: Beltz.

Horn, J. L. (1965). A rationale and test for the number of factors in factor analysis. Psychometrika, 30, 179-185. https://doi.org/ 10.1007/BF02289447

Inzlicht, M. \& Berkman, E. (2015). Six questions for the resource model of control (and some answers). Social and Personality Psychology Compass, 9, 511-524. https://doi.org/10.1111/ spc3.12200

Inzlicht, M. \& Legault, L. (2014). No pain, no gain: How distress underlies effective self-control (and unites diverse social psychological phenomena). In J. P. Forgas \& E. Harmon-Jones 
(Eds.), Sydney symposium of social psychology. Motivation and its regulation: The control within (pp. 115-132). New York, NY, US: Psychology Press.

Inzlicht, M. \& Schmeichel, B. J. (2012). What is ego depletion? Toward a mechanistic revision of the resource model of selfcontrol. Perspectives on Psychological Science, 7, 450-463. https://doi.org/10.1177/1745691612454134

Inzlicht, M. Schmeichel, B. J. \& Macrae, C. N. (2014). Why selfcontrol seems (but may not be) limited. Trends in Cognitive Sciences, 18, 127 -133. https://doi.org/10.1016/j.tics.2013.12. 009

Katzenbach, M., Blum, W., Drüke-Noe, C., Keller, K., Köller, O., Leiss, D. et al. (2009). Bildungsstandards: Kompetenzen überprüfen. Mathematik Sekundarstufe I. (Heft 1 \& 2). Berlin: Cornelsen.

Kotabe, H. P. \& Hofmann, W. (2015). On integrating the components of self-control. Perspectives on Psychological Science, 10, 618-638. https://doi.org/10.1177/1745691615593382

Kühnel, J., Syrek, C. J. \& Dreher, A. (2018). Why don't you go to bed on time? A daily diary study on the relationships between chronotype, self-control resources and the phenomenon of bedtime procrastination. Frontiers in Psychology, 9, 77. https://doi.org/ 10.3389/FPSYG.2018.00077

Kwan, M. Y. W., Bedard, C., King-Dowling, S., Wellman, S. \& Cairney, J. (2016). MovingU: A prospective cohort study to understand behavioural and environmental contexts influencing physical activity during the transition into emerging adulthood. BMC Public Health, 16, 728. https://doi.org/10.1186/s12889016-3372-7

Lian, H., Yam, K. C., Ferris, D. L. \& Brown, D. (2017). Self-control at work. Academy of Management Annals, 11, 703-732. https:// doi.org/10.5465/annals.2015.0126

Lindner, C., Nagy, G., Ramos Arhuis, W. A. \& Retelsdorf, J. (2017). A new perspective on the interplay between self-control and cognitive performance: Modeling progressive depletion patterns. PLOS ONE, 12, e0180149. https://doi.org/10.1371/journal.pone. 0180149

Lindner, C., Nagy, G. \& Retelsdorf, J. (2015). The dimensionality of the Brief Self-Control Scale-An evaluation of unidimensional and multidimensional applications. Personality and Individual Differences, 86, 465-473. https://doi.org/10.1016/j.paid.2015. 07.006

Lindner, C., Nagy, G. \& Retelsdorf, J. (2018). The need for self-control in achievement tests: Changes in students' state self-control capacity and effort investment. Social Psychology of Education, 21, 1113-1131. https://doi.org/10.1007/s11218-018-9455-9

Little, T. D., Preacher, K. J., Selig, J. P. \& Card, N. A. (2007). New developments in latent variable panel analyses of longitudinal data. International Journal of Behavioral Development, 31, 357 365. https://doi.org/10.1177/0165025407077757

Little, T. D., Slegers, D. W. \& Card, N. A. (2006). A Non-arbitrary method of identifying and scaling latent variables in SEM and MACS models. Structural Equation Modeling: A Multidisciplinary Journal, 13, 59-72. https://doi.org/10.1207/s15328007sem 1301_3

Lurquin, J. H. \& Miyake, A. (2017). Challenges to ego-depletion research go beyond the replication crisis: A need for tackling the conceptual crisis. Frontiers in Psychology 8, 568. https://doi. org/10.3389/fpsyg.2017.00568

Marsh, H. W. (2007). Application of confirmatory factor analysis and structural equation modeling in sport and exercise psychology. In G. Tenenbaum \& R. C. Eklund (Eds.), Handbook of Sport Psychology (3rd ed., pp. 774-798). Hoboken, NJ: Wiley. https://doi.org/10.1002/9781118270011.ch35

Marsh, H. W. \& Hau, K.-T. (1996). Assessing goodness of fit. Is parsimony always desirable? The Journal of Experimental Ed- ucation, 64, 364 - 390. https://doi.org/10.1080/00220973.1996. 10806604

McArdle, J. J. (1988). Dynamic but structural equation modeling of repeated measures data. In J. R. Nesselroade \& R. B. Cattell (Eds.), Handbook of multivariate experimental psychology (pp. 561 -614). Springer, Boston, MA. https://doi.org/10.1007/ 978-1-4613-0893-5_17

Musch, J., Brockhaus, R. \& Bröder, A. (2002). Ein Inventar zur Erfassung von zwei Faktoren sozialer Erwünschtheit. Diagnostica, 48, 121 -129. https://doi.org/10.1026//0012-1924.48.3.121

Muthén, L. \& Muthén, B. (2017). Mplus user's guide (8th ed.). Los Angeles: Authors.

Podsakoff, P. M., MacKenzie, S. B., Lee, J. Y. \& Podsakoff, N. P. (2003). Common method biases in behavioral research: A critical review of the literature and recommended remedies. Journal of Applied Psychology, 88, 879 - 903. doi: https://doi.org/10. 1037/0021-9010.88.5.879

Prem, R., Kubicek, B., Diestel, S. \& Korunka, C. (2016). Regulatory job stressors and their within-person relationships with ego depletion: The roles of state anxiety, self-control effort, and job autonomy. Journal of Vocational Behavior, 92, 22 - 32. https:// doi.org/10.1016/j.jvb.2015.11.004

Rivkin, W., Diestel, S. \& Schmidt, K.-H. (2014). The positive relationship between servant leadership and employees' psychological health: A multi-method approach. German Journal of Human Resource Management: Zeitschrift für Personalforschung, 28, 52-72. https://journals.sagepub.com/doi/pdf/10. $1177 / 239700221402800104$

Rivkin, W., Diestel, S. \& Schmidt, K.-H. (2015). Affective commitment as a moderator of the adverse relationships between dayspecific self-control demands and psychological well-being. Journal of Vocational Behavior, 88, 185-194. https://doi.org/ 10.1016/j.jvb.2015.03.005

Rowland, Z., Wenzel, M. \& Kubiak, T. (2016). The effects of computer-based mindfulness training on self-control and mindfulness within ambulatorily assessed network systems across health-related domains in a healthy student population (SMASH): Study protocol for a randomized controlled trial. Trials, 17, 570. https://doi.org/10.1186/s13063-016-1707-4

Salmon, S. J., De Vet, E., Adriaanse, M. A., Fennis, B. M., Veltkamp, M. \& De Ridder, D. T. D. (2015). Social proof in the supermarket: Promoting healthy choices under low self-control conditions. Food Quality and Preference, 45, 113-120. https://doi.org/10. 1016/j.foodqual.2015.06.004

Schmeichel, B. J., Vohs, K. D. \& Baumeister, R. F. (2003). Intellectual performance and ego depletion: Role of the self in logical reasoning and other information processing. Journal of Personality and Social Psychology, 85, 33 -46. https://doi.org/10. 1037/0022-3514.85.1.33

Schöndube, A., Bertrams, A., Sudeck, G. \& Fuchs, R. (2017). Selfcontrol strength and physical exercise: An ecological momentary assessment study. Psychology of Sport and Exercise, 29, 19 - 26. https://doi.org/10.1016/J.PSYCHSPORT.2016.11.006

Spearman, C. (1904). "General Intelligence", objectively determined and measured. The American Journal of Psychology, 15, 201 - 292. https://doi.org/10.2307/1412107

Steenkamp, J.-B. E. M. \& Baumgartner, H. (1998). Assessing measurement invariance in cross-national consumer research. Journal of Consumer Research, 25, 78-90. https://doi.org/10. $1086 / 209528$

Stöber, J. (1999). Die Soziale-Erwünschtheits-Skala-17 (SES-17): Entwicklung und erste Befunde zu Reliabilität und Validität. Diagnostica, 45, 173-177. https://doi.org/10.1026//0012-1924. 45.4.173

Tangney, J. P., Baumeister, R. F. \& Boone, A. L. (2004). High selfcontrol predicts good adjustment, less pathology, better grades, 
and interpersonal success. Journal of Personality, 72, 271-324. https://doi.org/10.1111/j.0022-3506.2004.00263.x

Tice, D. M., Baumeister, R. F., Shmueli, D. \& Muraven, M. (2007). Restoring the self: Positive affect helps improve self-regulation following ego depletion. Journal of Experimental Social Psychology, 43, 379-384. https://doi.org/10.1016/j.jesp.2006.05.007

Welsh, D. T. \& Ordóñez, L. D. (2014). The dark side of consecutive high performance goals: Linking goal setting, depletion, and unethical behavior. Organizational Behavior and Human Decision Processes, 123, 79 - 89. https://doi.org/10.1016/J.OBHDP. 2013.07.006

\section{Historie}

Onlineveröffentlichung: 06.06.2019

\section{ORCID}

Christoph Lindner

(iD) https://orcid.org/0000-0001-5688-3146

\section{Dr. Christoph Lindner}

\section{Dr. Marlit Annalena Lindner}

Leibniz-Institut für die Pädagogik der Naturwissenschaften und Mathematik

Erziehungswissenschaft und Pädagogische Psychologie

Olshausenstraße 62

24118 Kiel

clindner@ipn.uni-kiel.de

Christian-Albrechts-Universität zu Kiel

Referat für Lehrerbildung

Kuhnkestraße 2

24118 Kiel

Prof. Dr. Jan Retelsdorf

Universität Hamburg

Fakultät für Erziehungswissenschaft

Von-Melle-Park 8

20146 Hamburg 